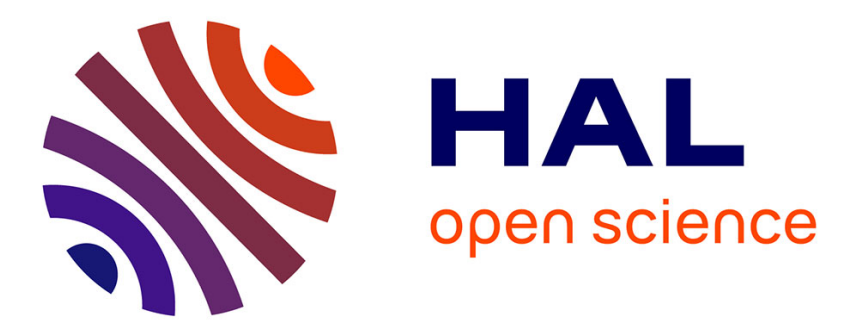

\title{
Toward a Context-Aware and Automatic Evaluation of Elderly Dependency in Smart Homes and Cities
}

\author{
Tayeb Lemlouma, Sébastien Laborie, Philippe Roose
}

\section{To cite this version:}

Tayeb Lemlouma, Sébastien Laborie, Philippe Roose. Toward a Context-Aware and Automatic Evaluation of Elderly Dependency in Smart Homes and Cities. The Fourteenth International Symposium on a World of Wireless, Mobile and Multimedia Networks (WoWMoM/SCUCA), Jun 2013, Madrid, Spain. pp.WoWMoM/SCUCA. hal-00839661

\section{HAL Id: hal-00839661 https://hal.science/hal-00839661}

Submitted on 11 Jun 2016

HAL is a multi-disciplinary open access archive for the deposit and dissemination of scientific research documents, whether they are published or not. The documents may come from teaching and research institutions in France or abroad, or from public or private research centers.
L'archive ouverte pluridisciplinaire HAL, est destinée au dépôt et à la diffusion de documents scientifiques de niveau recherche, publiés ou non, émanant des établissements d'enseignement et de recherche français ou étrangers, des laboratoires publics ou privés. 


\section{Toward a Context-Aware and Automatic Evaluation of Elderly Dependency in Smart Homes and Cities}

\author{
Tayeb Lemlouma \\ IRISA Lab - University of Rennes 1 \\ Lannion, France \\ tayeb.lemlouma@irisa.fr
}

\author{
Sébastien Laborie, Philippe Roose \\ LIUPPA - T2i \\ Anglet, France \\ \{sebastien.laborie, philippe.roose\}@univ-pau.fr
}

\begin{abstract}
In this work, we propose a framework for automatic dependency evaluation that can notify the changes of the elderly dependency, hence providing persons with required services in time. In order to validate our approach, we target the French dependent population. Especially, we focus our proposal on the AGGIR dependency model used in the French medical field. Our objective is to provide a flexible architecture and an extensible model linked to opened data that can refer to a wide variety of services (hardware sensors and software; simple or composed services).
\end{abstract}

Keywords- Dependency; activities of daily living; smart home; smart city; services; service composition

\section{INTRODUCTION}

According to the United Nations projections [1], in 2050, the old-age dependency ratio of the population aged 65 years or over will approximate $51,70 \%$ of the population aged 20 64. For instance, this ratio will approximate $48,2 \%$ in France, $62 \%$ in Germany and $44,10 \%$ in UK (Fig. 1). In healthcare domain, the evaluation of the autonomy or dependency of a person is of high importance. Indeed, such evaluation is used by the professionals of health to identify the person's needs of assistance, services and allowance. Also, it allows taking the right decision about keeping the person in the health institution, nursing home or independently at home with or without healthcare monitoring. In a smart home environment, and more globally in a smart city environment, particular attention and care should be made for dependent people, in particular for elderly since they are left on their own at home or in the city. A continuous dependency evaluation is of high concern since it can detect the changes regarding the person's abilities to achieve elementary daily tasks. In this work, we propose a framework for automatic and flexible dependency evaluation that can notify any changes of the elderly dependency and hence allows providing him, in time, with required help, assistance and allowance. We consider the heterogeneity of elderly profiles and service sources that can come from anywhere from the home or the city. The dependency evaluation takes benefits from the description model of the elderly and the dynamic composition of services. Our approach considers the activities of daily living (ADL) as used in the French AGGIR (Autonomy Gerontology Iso-Resources Group) model [2]. The automatic way in observing the different elderly activities allows avoiding human errors and patient's ignorance that face the current use of the AGGIR tool and similar evaluation methods used in geriatrics.

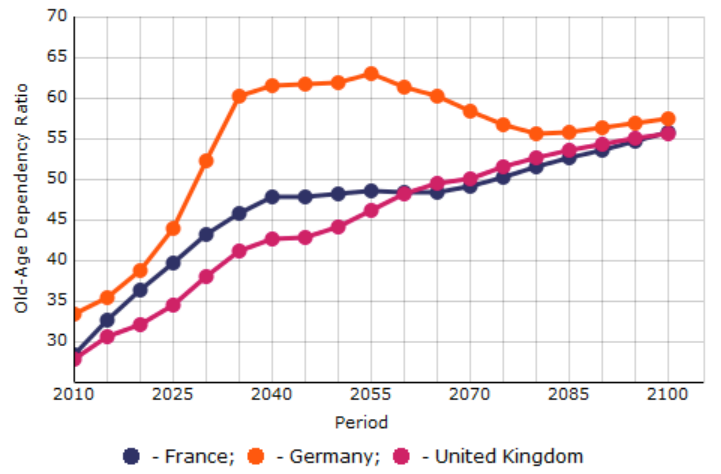

Figure 1. Old-Age Dependency Ratio in France, Germany and UK

\section{RELATED WORK}

The person's dependency can be defined as the ability of a person to achieve elementary tasks of daily living without the help or stimulation of a third party. In the geriatrics domain, different methods and tools have been defined to evaluate this dependency using different factors, such as age, medical status, mobility, fall risk, etc. The main reason of the existence of such different methods is the determination of what is a basic activity of daily living (ADL, called also BADL). Methods are mostly based on the ADL definitions of Katz [3], Barthel [4] and the instrumental daily activities (IADL) of Lawton [5]. Among these very numerous scales, we cite for instance RAI [6], AGGIR, SMAF [7] and MOS SF-36 [8]. In the evaluation tools, the notion of group is used to define a set of persons with common characteristics in terms of needs to healthcare assistance and help. For instance, we find the Diagnosis Related Groups (DRG) in USA [9] and the iso-resource group (GIR) in France [2].

We notice that most of these evaluation methods are achieved using questionnaires and manual scales, and are consequently subject of human errors or patients ignorance. Various research efforts, like [10][11][12][13][14], investigate the automatic (or semi-automatic) context aware monitoring of elderly activities.

In [10], an OSGi-based infrastructure is proposed for smart homes in order to build context-aware services based on an ontology model. A middleware (SOCAM) was presented and includes services that perform context 
discovery, acquisition and interpretation. In the proposed architecture, the required intensive computation delays (due to the logic reasoning and context processing) are inconvenient in the context of providing services to dependent persons. Furthermore, it is not easy to integrate heterogeneous services provided by third party. Le et al. presented in [11] a system for activity recognition using a set of presence sensors. The considered activity is limited to the detection of mobile and immobile states of elders. Immobile states are identified from the observation of elder's daily life.

The study in [12] focuses on the prevention of ADL dependency through models of elderly dependency risk. The analysis of models is based on the interview of an important set of self-respondents with different interview models including cognitive measures. The work in [25] discussed fall detection and identification using a new algorithm based on visual cues that exploits robust and stable foreground object tracking methods. In [13], the authors propose a method using sensors for detecting abnormal changes in the resident's behavior. It focuses on the detection and quantification of a potential nycthemeral shift in activity through a statistical approach. The work is based on infrared sensors placed in each room of the resident's home. The work in [14] describes a system that monitors elderly in order to automatically detect signs of illness. The system is based on an early illness detection using a set of medical sensors that report data for visualization and interpretations by healthcare professionals, such as nurses and doctors. The considered environment by the proposed system is a clinicallike system that focuses on significant health events. These characteristics make difficult and less flexible the integration of the system in a home or a city-like environment.

Our approach aims to make the use of the dependency evaluation methods automatic and more flexible. This is done thanks to the ability of including any kind of services and observation sources. Despite the specification of services per daily activity that we will identify in order to validate our architecture, we do not make any restriction neither on the kind of the used services (hardware sensors, software or manual inputs) nor in data sources used in handling the elderly dependency. Our framework takes benefits from the collaboration of these different sources and opened technologies that facilitate the processing of heterogeneous services and sources. Our proposed approach considers the main activities of daily living as a whole and does not focus on a particular activity like some previous work. This choice is done because of the heterogeneity of the monitored elderly persons; also, we believe that when a dependency degree changes, this is the consequence of not only one activity of the person but also several of them. Consequently, such changes should be quickly notified to healthcare providers.

\section{CONTEXT Aware AND Automatic Evaluation of DEPENDENCY}

Most of context-aware approaches refer to the Dey's context definition [15] as any information that can be used to characterize the situation of an entity. An entity includes a person, a place or an object. This work focuses on the evaluation of the dependency in order to help healthcare professionals to provide services and monitoring without keeping persons in the health institution. Hence, the context considered in this work is represented by a set of the different variables that influence the elderly dependency evaluation. In order to validate our approach, we target the French dependent population and in particular elderly dependent persons. In France, in order to evaluate the needs of an elderly person in terms of medical assistance and allowance, the AGGIR model is used [2].

Like other dependency evaluation scales, the AGGIR evaluation is achieved manually by a medical reviewer, such as the referring doctor or a medical assistant. Each daily activity of a dependent person is qualified by the medical reviewer using the four possible adverbs: Spontaneously $(S)$, Completely/Totally $(T)$, Usually $(U)$ and Correctly $(C)$. The $S$ adverb means that activity can be done without any stimulation. The $T$ adverb means that all of the actions involved in the activity can be done by the person. The $U$ adverb means that the activity is done regularly; the frequency of the execution depends to the nature of the activity. The $C$ adverb involves the quality of person's realization, safety and conformance with the recommended usage of the activity. According to a logical condition involving these adverbs, the activity is evaluated with the modalities: $A, B$ or $C$. A given activity is evaluated with the modality $A$, if the person can achieve the activity with the following condition: $S \wedge T \wedge C \wedge U$; the condition of the $C$ evaluation is: $\neg S \wedge \neg T \wedge \neg C \wedge \neg U$; the $B$ evaluation is given if the previous conditions are not satisfied. The $A$ evaluation means that the person is completely autonomous in achieving the given activity, $B$ means that the person is partially dependent and $C$ means that the person is dependent and cannot achieve the activity alone.

The AGGIR model considers 17 variables describing the activities of daily living, such as the mental coherence, hygiene and mobility. Each variable evaluates the dependency degree of achieving a given activity. Based on a multivariate analysis involving more than 5000 persons, the model has identified 10 discriminated variables, 8 of them are used in the classification of the dependent persons into 13 profile ranks and 6 groups. The 13 profile ranks concern the losses of autonomy of dependent persons. The 6 groups, called iso-resource groups or GIR, reduce the number of profile ranks and concern the needs of assistance and allowances. The GIR algorithm computes the iso-resource group number (1 to 6) based on predefined association between profile ranks and groups. For instance, the rank 1 is associated to the GIR 1, ranks from 2 to 7 are associated to the GIR 2, etc. (Table I.). The first group (GIR 1) represents the persons that are completely dependent while the last group (GIR 6) represents autonomous persons.

To identify the profile rank of a person, the model uses 8 classification functions that compute the classification scores. The person is classified as belonging to the profile rank for which he/she has the highest classification score (Table I.). Scores conditions are tested in a sequential order of the classification functions from $S_{1}$ to $S_{8}$. Classification functions are defined as: $S_{\mathrm{i}}=\sum_{k=1}^{8} w_{i k}$, where, $S_{\mathrm{i}}$ is the score 
of the $i$ 'th function; $w_{\mathrm{ij}}$ is the weight for the $j^{\prime}$ th variable modality (i.e., $A, B$ or $C$ ).

TABLE I. ASSOCIATION BETWEEN PROFILE RANKS, ClASSIFICATION SCORES AND GIR

\begin{tabular}{|c|c|c|}
\hline Profile Ranks & Score Condition & GIR \\
\hline 1 & $S_{1} \geq 4380$ & 1 \\
\hline 2 & $4140 \leq S_{1}<4380$ & \\
3 & $3390 \leq S_{1}<4140$ & \\
4 & $S_{2} \geq 2016$ & 2 \\
5 & $S_{3} \geq 1700$ & \\
6 & $1432 \leq S_{3}<1700$ & \\
7 & $S_{4} \geq 2400$ & \\
\hline 8 & $S_{5} \geq 1200$ & 3 \\
9 & $S_{6} \geq 800$ & \\
\hline 10 & $S_{7} \geq 650$ & 4 \\
\hline 11 & $S_{8} \geq 4000$ & \\
\hline 12 & $2000 \leq S_{8}<4000$ & 5 \\
\hline 13 & $S_{8}<2000$ & 6 \\
\hline
\end{tabular}

Table II presents the different weights of the different variables modalities regarding $S_{1}$ and $S_{2}$ functions. Let us consider the example of a person with the following evaluation: Coherence $=C$, Orientation $=C$, Hygiene $=C$, Dressing $=A$, Eating $=C$, Elimination $=A$, Transferts $=A$ and Interior Moving $=B$. We have $S_{1}=\sum_{k=1}^{8} w_{1 k}=3332$ and $S_{2}=\sum_{k=1}^{8} w_{2 k}=2760$ (Table II.). To identify the profile rank, the score conditions are tested first with the $S_{1}$ score, if there is no satisfied condition, the score is then tested with $S_{2}$ and so forth until the last condition of $S_{8}\left(S_{8}<2000\right)$. Here, the score of $S_{1}$ (3332) does not satisfy the $S_{1}$ score conditions (Table I.). However, the score of $S_{2}$ (2760) satisfies the " $S_{2} \geq$ 2016" condition (Table I). Hence, the person's profile rank is 4 and his iso-resource group is 2 .

TABLE II. WEIGHTS OF THE CLASSIFICATION FUNCTIONS $S_{1}$ AND $S_{2}$

\begin{tabular}{|c|c|c|c|l|c|c|c|}
\hline Activity & & $\boldsymbol{W}_{\mathbf{1 i}}$ & $\boldsymbol{W}_{\mathbf{2 i}}$ & & & $\boldsymbol{W}_{\mathbf{1 i}}$ & $\boldsymbol{W}_{\mathbf{2 i}}$ \\
\hline Coherence & $\mathrm{C}$ & 2000 & 1500 & Eating & $\mathrm{C}$ & 60 & 60 \\
\hline & $\mathrm{B}$ & 0 & 320 & & $\mathrm{~B}$ & 20 & 0 \\
\hline & $\mathrm{A}$ & 0 & 0 & & $\mathrm{~A}$ & 0 & 0 \\
\hline Orientation & $\mathrm{C}$ & 1200 & 1200 & Elimination & $\mathrm{C}$ & 100 & 100 \\
\hline & $\mathrm{B}$ & 0 & 120 & & $\mathrm{~B}$ & 16 & 16 \\
\hline & $\mathrm{A}$ & 0 & 0 & & $\mathrm{~A}$ & 0 & 0 \\
\hline Hygiene & $\mathrm{C}$ & 40 & 40 & Transferts & $\mathrm{C}$ & 800 & 800 \\
\hline & $\mathrm{B}$ & 16 & 16 & & $\mathrm{~B}$ & 120 & 120 \\
\hline & $\mathrm{A}$ & 0 & 0 & & $\mathrm{~A}$ & 0 & 0 \\
\hline Dressing & $\mathrm{C}$ & 40 & 40 & Int. Moving & $\mathrm{C}$ & 200 & -80 \\
\hline & $\mathrm{B}$ & 16 & 16 & & $\mathrm{~B}$ & 32 & -40 \\
\hline & $\mathrm{A}$ & 0 & 0 & & $\mathrm{~A}$ & 0 & 0 \\
\hline
\end{tabular}

We propose to encode inside a person profile the required information that concerns the patient itself (i.e., name, address, age, etc.) and all the values (i.e., $A, B$ and $C$ ) that correspond to the 8 evaluation variables. Of course, each time a new evaluation of a person is made, we aggregate the results in the profile and attach to it a specific date in order to confront different evaluations, thus sending notifications if needed. Figure 2 shows an example of a person profile that corresponds to a potential instance of an evaluation process. The beginning of the profile shows details about the patient. In the illustrated example, the profile is related to a person named John Doeuf, which is 55 years old. Thereafter, for a specific date, some values of the evaluation variables are detailed.

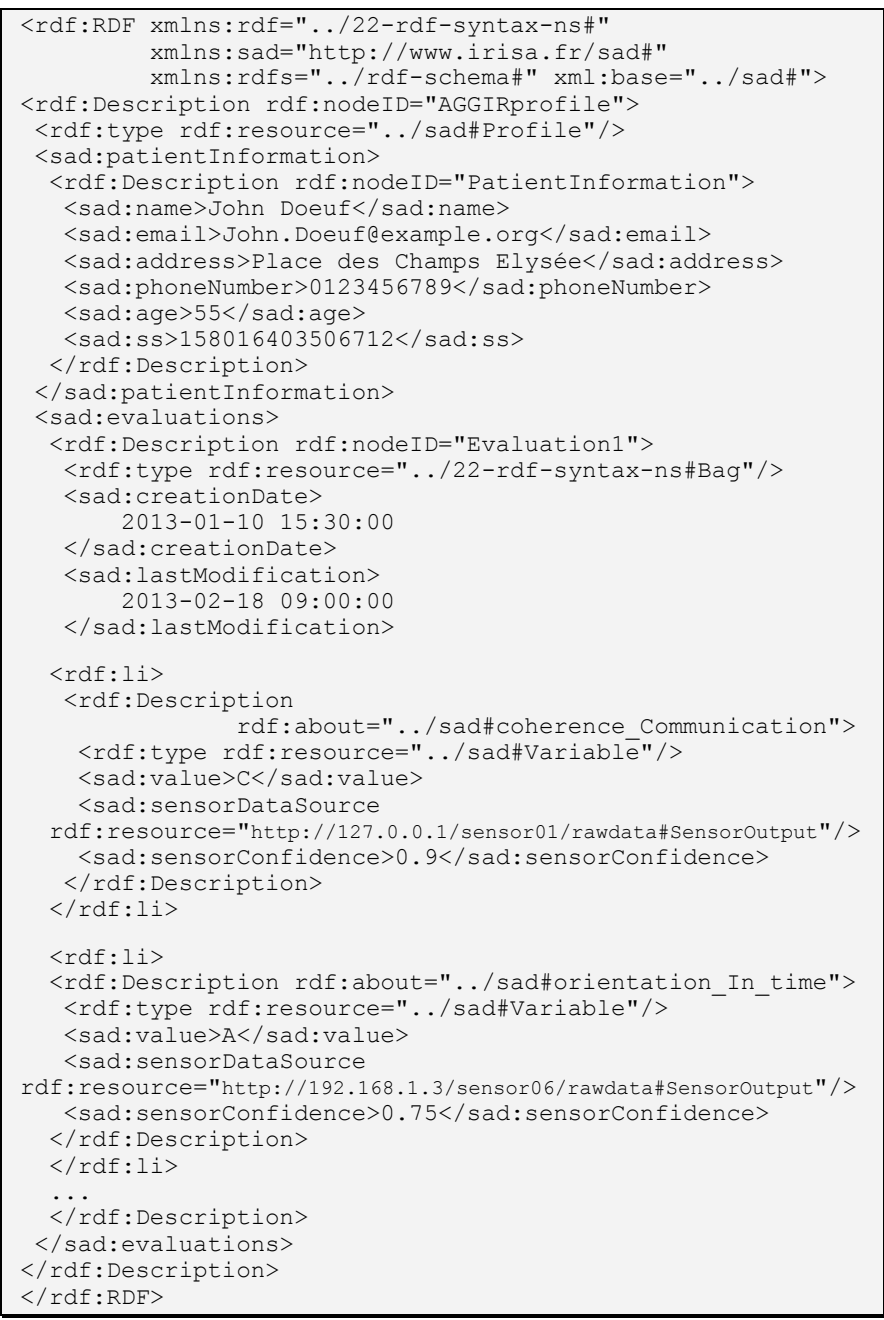

Figure 2. An Example of a Person Profile Encoded in RDF/XML

As illustrated in the figure above, we have proposed an $\mathrm{RDF} / \mathrm{XML}$ serialization [16] of a person profile for the following reasons:

- RDF descriptions [17] allow us to easily perform aggregation of descriptions, which can be useful if several applications handle and maintain profile data that concerns the same person.

- RDF can handle semantic concepts (or vocabularies) described in ontologies, thus enhancing the person profile semantics. For instance, semantics allow us to state that a person living in Paris is also living in France. Hence, semantic queries searching information about persons living in France will retrieve some results, and especially those living in Paris.

- RDF links data from different sources. It is the core technology used by the Linked Data Community. Currently, billions of data available on the Web are interlinked with such a technology, e.g., DBpedia.

Since our objective in this paper is to automatically fill in the person profile (especially, for determining the output 
values of the 8 evaluation variables) thanks to services, we propose to enhance our profile model by referring to, for each variable, the service description and a confidence value. For instance, in Figure 2, the Coherence variable value (i.e., $C$ ) has been computed thanks to a specific service with a high confidence value, i.e., 0.9 (confidence values are set between 0 and 1 , the higher the value is, the higher is the confidence). Of course, thanks to the service URI, here http://127.0.0.1/sensor01/rawdata\#SensorOutput, it is possible to access to other descriptions that concern the service details, e.g., the service characteristics, the service location, the service owner, etc.

Based on the person profiles, we have performed the simulations of all the possible activities evaluations within the six GIR groups. For instance, Figure 3 illustrates the ratio of each variable's modality in the iso-resources group 2 and 3. Our simulations have leaded us to identify the most characteristic daily activities of each dependency group. The objective is to identify what are the most likely activities that could be negatively affected when the dependency of a person changes. Consequently, given a dependency group of the person, some identified activities have to be given high priority in monitoring and observing them.

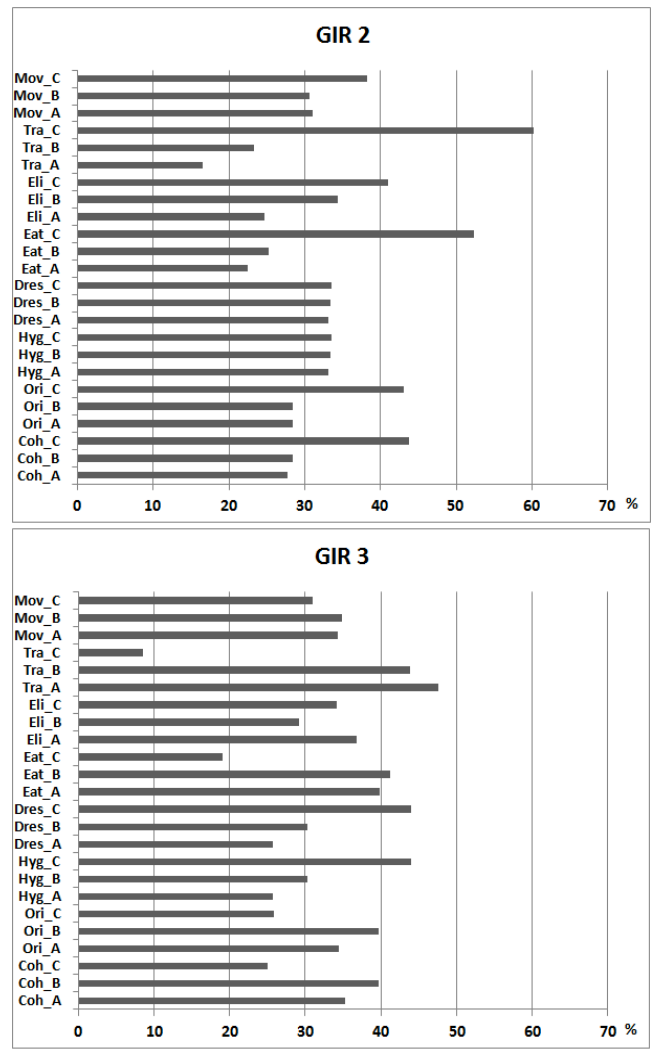

Figure 3. Variables Modalities Ratios in the Iso-resource Groups 2 and 3

From our simulations, we model the dependency changes with a transition graph between all of the 24 modalities ( 8 variables with the $A, B$ and $C$ modalities) from a group $N$ to a group $N-1$. Only the transition from a group $N$ to a group $N-1$ is considered because when a dependency change occurs, the current dependency group passes always first by the direct lower group. When a change in the dependency of a person occurs, this implies that the person is no longer able to achieve some daily activities. The new evaluation of such key activities should be negative (i.e., evaluated $B$ or $C$ ). Given a current dependency group $N$, in order to identify priority services for the detection of the dependency changes: (1) we only focus on the variables of the group $N-1$ with a negative modality ( $B$ or $C$ ) and a high ratio $\delta$ regarding the size of $N-1$ group's population, then (2) we keep only the transitions with an increased ration from a variable modality of the current group $N$ to the new group $N-1$. Figure 4 shows an overview of the results regarding the dependency change from the group 3 to 2 . If we fix the ration $\delta$ at $40 \%$, identified priority services will concern the following activities (Fig. 4): Transfers (a change with the modality $C$ from $8,58 \%$ to $60,16 \%$ ), Eating (modality $C$ from $19,04 \%$ to $52,28 \%$ ), Coherence (modality $C$ from $25 \%$ to $43,8 \%$ ) and Orientation (modality $C$ from $25,87 \%$ to $43,14 \%$ ).

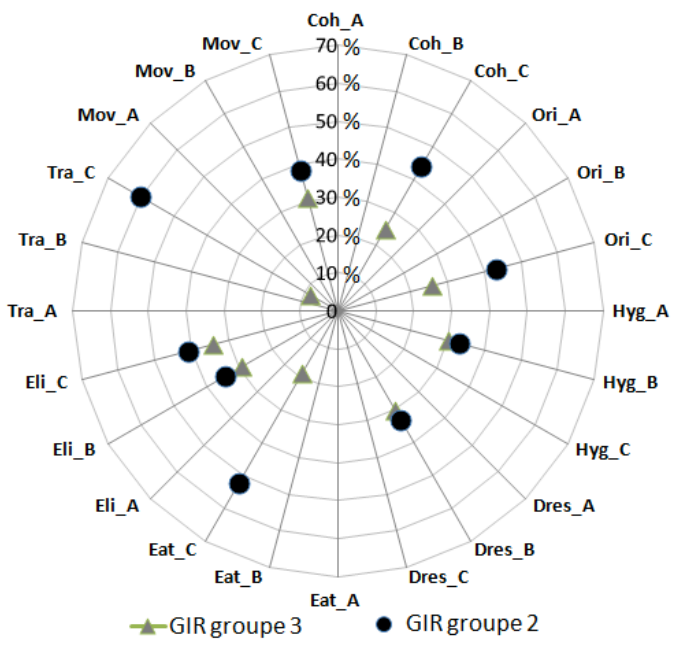

Figure 4. Dependency Change from the Group 3 to 2 .

\section{Proposed ARChitecture SPECIFICATION}

The use of traditional sensors (temperature, video, sound, presence, etc.) helps in detecting Activities of Daily Living (ADL) [18] (e.g., hygiene, toilet use, eating, resting, and dressing). In order to detect such ADL variables, it does not exist one unique sensor. Only a composition of several ones can provide an interesting measure. In the following part, we identify and review for each variable of the AGGIR model various configurations in order to detect and measure each variable in our architecture (Fig. 6).

\section{A. Variables:}

- Coherence: SF-36 Health Survey questionnaire [19] gives information and measure of current coherence. Such questionnaire can be easily designed using our already implemented platform for media services at home through the resident's TV set [24].

- Orientation: Floor plan and indoor GPS give orientation information and permit dedicated human to measure and/or detect troubles. 
- Hygiene: Ultrasonic water flow meter, sound detector, and flush switch. The use of ultrasonic water flow meter can measure the power and water consumption. The number of flushes may give adequate information. In [20], an automated bathroom activity monitoring system is based on the acoustics information.

- Dressing: Dressing door sensor aperture and IP Cam. Both can give an interesting measure of the quantitative use of the dressing. With human evolvement, the quantitative information can become qualitative in order to give information on the used clothes.

- Eating: light switch, door fridge sensor, hob sensors, mixer tap, etc. All those sensors can give information on the use of the kitchen.

- Elimination: Ultrasonic water flow meter, sound detector, flushes switch. The same sensors as the hygiene variable may also give information on elimination.

- Transfers: single inertial sensor. The use of inertial sensor-based motion analysis provides information in the longitudinal follow-up.

- Internal Moving: Floor plan and indoor GPS help in giving such information.

This non-exhaustive list of sensors, multi-sensors and human tasks analysis gives information of the amount of various possibilities for each variable. Moreover, some of them can be useful for several measures. Nevertheless, all those sensors and/or IS data or human activities are all heterogeneous. The heterogeneity is both on data format, contents and access mode (query, notify, regular, etc.).

Moreover, according to specific situations (sensor failure, emergency, mobility, etc.), they are not always available and may not give correct information. The use of dynamic composition can complete and give qualitative additional data (Fig. 6).

The following section presents a solution based on mediation in ambient environment and ADL oriented architecture with dynamic service composition.

\section{B. General Software Architecture}

The particularity of such application is its strong interaction with various heterogeneous devices and services. It raises two problems: a technological one because of communication interfaces (Bluetooth, Wi-Fi, Zigbee, proprietary, etc.) and a logical one (heterogeneity of data). Many existing frameworks have been proposed, we identify Cilia (Fig. 5), which is an open candidate to be the core of such heterogeneity management. "Cilia is a lightweight data mediation and integration service component model, built upon OSGi, the dynamic Java platform. Cilia provides a framework and toolset that simplify the development and the maintenance of large-scale, complex integration solutions that interacts with heterogeneous and complex integrated systems". Such ambient applications also need a dynamic composition of service (logical or physical).

Because of the mobility of users, the availability of devices, the energy consumption, and therefore economy, the orchestration of services aiming at providing high level tasks needs to continuously evolve in order to always being able to achieve its task.

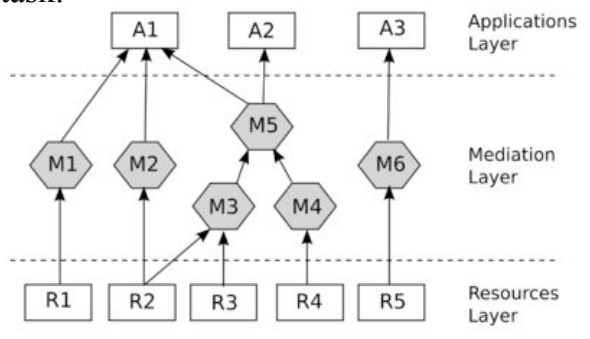

Figure 5. Cilia Mediation Architecture ${ }^{1}$ [21]

Moreover, the availability of services continuously increases and therefore, new available ones must be included in services compositions.

Each GIR variable (Coherence, Dressing, Int. moving, etc.) corresponds to a configuration. A configuration is an orchestration (done with BPMN [22][23]) of Cilia mediators, and each mediator will serve as a proxy for services in order to hide and solve heterogeneity problems (access, synchronization, data routing, etc.). The set of services, orchestrations and mediators forms the Architecture Layer.

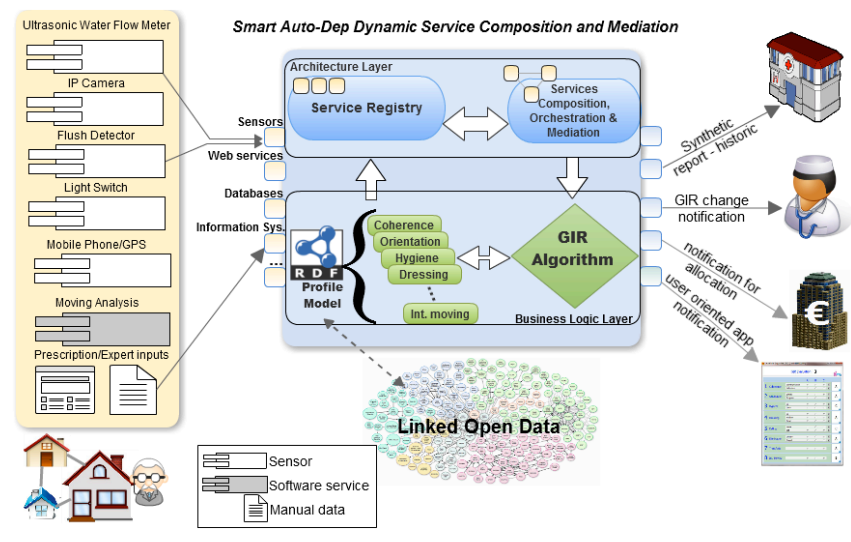

Figure 6. ADL oriented architecture

The result of each GIR service will be sent to the GIR Algorithm (also composed of atomic services in order to be dynamically adapted to different contexts and situations). Figure 7 presents our Smart Auto-Dep interface that implements the GIR algorithm and its extension with heterogeneous sources. Variables observations can come from heterogeneous data sources provided by different services (Fig. 6). The interface allows notifying the dependency change if it occurs. Notifications are based on a threshold value after which, if a dependency change is maintained, the notification is sent. The destination of the notification could be, for instance, the person's referring doctor or its medical assistance institution (hospital, clinic, etc., see Fig. 6). The rapid notification of the dependency change allows providing the elderly quick assistance and to consider its needs and allowance as soon as possible.

\footnotetext{
${ }^{1}$ http://wikiadele.imag.fr/index.php/Cilia
} 


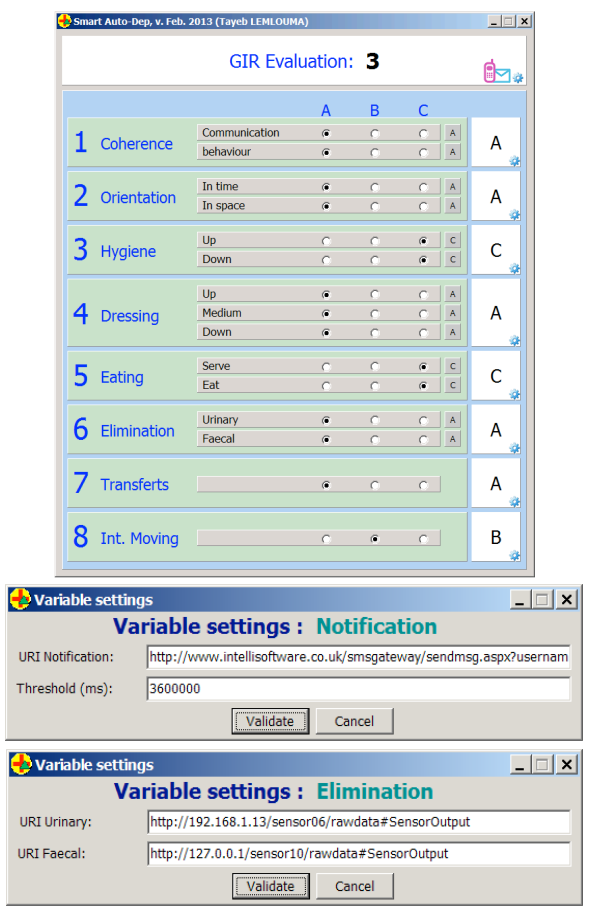

Figure 7. Smart Auto-Dep Interface

\section{CONCLUSION AND FUtURE WORK}

The objective of this paper was to make automatic the dependency evaluation process, which is usually done manually in the medical field. We focused on the dependency change within the medical AGGIR model. Our proposition tried to move the current situation toward a flexible architecture and an extensible model linked to opened data referring to a wide variety of services. We have identified priority variables for the dependency changes according to the context of the elderly (GIR groups). We described our vision of a flexible architecture that includes our implemented Smart Auto-Dep interface, which is ready to integrate various sources (services, sensors and data) and notify the dependency changes. Next steps will be to enrich our proposed RDF model, to explore other dependency models and to experiment the dependency changes with real heterogeneous sensors and services composition.

\section{REFERENCES}

[1] United Nations, Department of Economic and Social Affairs, http://esa.un.org/unpd/wpp/, acceded January 2013.

[2] AGGIR (Autonomy Gerontology Iso-Resources Group) model. "The national standardized instrument determining the attribution of the specific dependence allowance in France,' Minist. for Labour, Social Relations, the Family and Solidarity, Dec- 2008-821 (update of Dec2001-1084), Official J. of the French Gov.: JO of 21 august 2008.

[3] S. Katz and C. Akpom, "A measure of primary sociobiological functions," Intl. Journal of Health Services, vol. 6, no. 3, pp. 493508,1976 .

[4] F. Mahoney, D.W. Barthel, "Functional evaluation: the barthel index," Maryland State Medical Journal 14(2), pp. 61-5, 1965.

[5] M. Lawton, E. Brody, E., "Assessment of older people: Selfmaintaining and instrumental activities of daily living," Gerontologist, vol. 9, pp. 179-186, 1969.
[6] W. Achterberg, et al., "Het resident assessment instrument (rai): een overzicht van internationaal onderzoek naar de psychommetrische kwaliteiten en effecten van implementatie in verpleeghuizen," Tijdschrift Gerontologie en Geriatrie 30, 1999.

[7] R. Herbert, et al., "The functional autonomy measurement system (smaf): despcription and validation of an instrument for the measurement of handicaps", Age and Aging, vol. 17, pp. 293-302, 1988.

[8] M. Horney, "Measuring and monitoring general health status in elderly persons: practical and methodological issues in using the sf-36 health survey," Geronotologist, vol. 36, pp. 571-583, 1996.

[9] R. B. Fetter, Y. Shin, J.L. Freeman, et al. "Case mix definition by diagnosis related groups," Medical Care, vol. 18(2), pp. 1-53, 1980

[10] T. Gu, et al., "Toward an OSGi-Based Infrastructure for ContextAware Applications," IEEE CS and IEEE ComSoc, pp. 66-74, 2004.

[11] X. H. B. Le et al., "Health Smart Home - Towards an assistant tool for automatic assessment of the dependence of elders," IEEE Engineering in Medicine and Biology Society (EMBS 2007), Aug. 2007, pp. 3806-3809, doi:10.1109/IEMBS.2007.4353161.

[12] D.O.C Thomas, et al., "Improving the Validity of Activity of Daily Living Dependency Risk Assessment," Journal of Applied Gerontology, January 17, 2013, doi: 10.1177/0733464812471894

[13] C. Franco, et al., "Behavioral Telemonitoring of the Elderly at Home: Detection of Nycthemeral Rhythms Drifts from Location Data," In Proc. of the 24th IEEE International Conference on Advanced Information Networking and Applications Workshops (WAINA), pp. 759-766, April 2010, doi: 10.1109/WAINA.2010.81

[14] J. Rantz Marilyn J. et al. "Automated technology to speed recognition of signs of illness in older adults," J. of Gerontological Nursing, Vol. 38(4): pp. 18-23, April 2012; doi:10.3928/00989134-20120307-01

[15] A. Dey, "Understanding and Using Context," Personal and Ubiquitous Computing Journal, Vol 5, Issue 1, pp. 4-7, 2001.

[16] D. Beckett. "RDF/XML Syntax Specification (Revised)". W3C Rec., 10 Feb. 2004. http://www.w3.org/TR/REC-rdf-syntax/

[17] F. Manola and Eric Miller, "RDF Primer", W3C Recommendation, 10 February 2004. http://www.w3.org/TR/rdf-primer/

[18] L. Monaci and R.G Morris, "Neuropsychological screening performance and the association with activities of daily living and instrumental activities of daily living in dementia: baseline and 19 - to 24 - month follow-up," Int. Journal of Geriatric Psychiatry, vol 27:2, 2012, pp.197-204

[19] B. Nygrena, et al., "Resilience, sense of coherence, purpose in life and self-transcendence in relation to perceived physical and mental health among the oldest old," Aging \& Mental Health - Vol. 9, Issue 4, 2005 pp. 354-362 - DOI:10.1080/1360500114415

[20] J. Chen, et al., "Bathroom activity monitoring based on sound", In Proceedings of PERVASIVE 2005, pp. 47-61, 2005

[21] I. Noé G. et al. "A Reflective Framework for Mediation Applications," In Proc. of the 10th Int. Middleware Workshop on Adaptive and Reflective Middleware, 2011-12-12, Lisbon, Portugal

[22] Z. Hangjung, et al., "End-to-end reliability of service oriented applications," Journal Information Systems Frontiers, Vol. 14:5, Kluwer Academic Publishers Hingham, USA, pp. 971-986, 2012.

[23] M. Krizevnik, M. B. Juric - "Data-bound variables for WS-BPEL executable processes", Journal Computer Languages, Systems and Structures archive Volume 38 Issue 4, December, 2012 Pages 279299 - Elsevier Science Publishers B. V. Amsterdam, The Netherlands, The Netherlands

[24] T. Lemlouma and A. M. Chalouf, "Smart Media Services Through TV Sets for Elderly and Dependent Persons," Springer-IEEE/EMB Int. Conf. on Wireless Mobile Communication and Healthcare (MOBIHEALTH'12), Paris, France, 2012.

[25] M. Kokkinos, N. Doulamis and A. Doulamis, "Local Geometrically Enriched Mixtures for Stable and Robust Human Tracking in Detecting Falls," International Journal of Advanced Robotic Systems, DOI: 10.5772/54049, 2013. 